\title{
Good continuation affects visual detection
}

\author{
WILLIAM PRINZMETAL \\ Claremont Graduate School, Claremont, California 91711 \\ and \\ WILLIAM P. BANKS \\ Pomona College and Claremont Graduate School, Claremont, California 91711
}

\begin{abstract}
This research shows that the principle of good continuation can predict forced-choice detection of an element imbedded in a briefly presented visual array. The present experiments show the configurational effect in a manner that is difficult for feature-analytic theories to explain. The research adds good continuation to good form as Gestalt-like organizational principles affecting detection performance, and it shows the necessity for including organizational principles in a theory of visual detection.
\end{abstract}

The present study compares Banks and Prinzmetal's (1976) configurational hypothesis of visual detection to feature analytic approaches, of which the interactive channels theory (Estes, 1972, 1974) is the most complete. Both the interactive channels theory and the configurational hypothesis can explain the major findings in visual detection experiments. This study tests the two theories in a detection paradigm experiment where they do not make the same prediction.

In the detection paradigm, a subject must choose which of a small set of prespecified target items appeared in a briefly presented stimulus array. Four results of this paradigm are extremely reliable and important. The first is a decline in detection accuracy as the number of noise items in the array increases (Estes \& Taylor, 1966). This is generally referred to as the display-size effect. Second, performance declines as the similarity between target and noise (nontarget) items in the array increases (Estes, 1972; Gardner, 1973; McIntyre, Fox, \& Neale, 1970). Third, performance improves as similarity among noise items increases (Estes, 1974; McIntyre et al. 1970; McLaughlin, Masterson, \& Herrmann, 1972). Finally, target detection is inversely related to targetnoise proximity (Banks, Bodinger, \& Illige, 1974; Estes, 1972; Eriksen \& Eriksen, 1974).

Estes' interactive channels theory postulates input channels located about the visual field that feed into feature detectors. Information input through differ-

This research was supported by National Science Foundation Grant BMS 75-20328. The authors wish to thank Julie Flora, who helped conduct some preliminary investigations that led eventually to this study, and the Claremont Cognitive Lunch group for their constructive criticism. This study was presented at the Psychonomics Society annual meeting, St. Louis, Missouri, November 1976. Requests for reprints can be sent to either author at the Department of Psychology, Pomona College, Claremont, California 91711 . ent channels to any feature detector is mutually inhibitory. As target-noise similarity and proximity increase, the probability of activating channels connected to the same feature detector increases, mutual inhibition takes place, and the effects of target-noise similarity and proximity are predicted. The display size effect results from the increased probability that a confusable noise item will inhibit an input channel for the target when the density of elements in the display is increased. Estes (1974) explains the effect of noise homogeneity in terms of two separate effects of the variability of features in the noise. Increasing variability (i.e., decreasing homogeneity) increases the probability that a noise element will be confused with a target; increasing variability also increases the probability of inhibitory interactions among input channels to feature detectors. Estes (1974) has separate mathematical appendices showing how each of these effects can take place.

The configurational approach assumes that groups of items are organized by the perceptual system in a prerecognition stage analogous to Kahneman's (1973) unit formation stage or Kinchla's (1974) elementary coding stage. The groups would be defined according to principles similar to the Gestalt laws. Predictions of performance in target detection are based on the degree to which the target merges with or stands out from the noise of elements as a result of the work of the unit formation stage. If the target items form a perceptual group with the noise elements, detection performance will be worse than when the target does not group with noise elements, but is processed separately. Since perceptual grouping depends on such factors as similarity, proximity, good form, good continuation, and other Gestaltlike principles, the four basic effects follow naturally. If target and noise items are similar to each other or 
are located near each other, they will group by the principles of similarity and proximity grouping, respectively, and this grouping will degrade performance. If noise items are homogeneous, they will tend to group together and separately from the target item, improving performance. The display size effect results from the increased target-noise grouping that occurs as more noise items are crowded into the display.

In order for the configurational approach to make predictions that are different from those of the interactive channels approach, the effect of organizational principles other than similarity or proximity must be demonstrated. Banks and Prinzmetal (1976) used goodness of form as a configurational principle that feature-analytic theories such as Estes' seemed unable to account for. Banks and Prinzmetal found that when the target element was placed in an array of noise such that it formed a "good" figure with them, detection performance was worse than when additional noise elements were put in the array to destroy the good figure. However, the configurations used in that experiment are open to the interpretation that the noise elements in the "bad" form could mutually inhibit each other and reduce their interfering effect on the target. This is an ad hoc explanation since interference between noise items has not previously been invoked by feature analytic theories, but it still could be correct.

The present study uses the classic grouping principle of good continuation to create detection arrays in which the configurational theory and the feature analytic approach make different predictions. All the present arrays contain a "line" of noise items. The configurational theory predicts that when the target appears to be part of a line, it should group with the noise items and be detected more poorly than when it does not appear to be part of the line. We constructed our arrays according to Prytulak's (1974) empirical principles of good continuation grouping so that our basis for predicting grouping effects would be empirical rather than intuitive. To make a clear distinction between the prediction of our model and a feature-interference model, the arrays were always constructed with the target closer, on the average, to noise items when it was not grouped with the line of noise items than when it was.

Both Experiments 1 and 2 are two alternative forced-choice detection experiments. Experiment 1 is a straightforward experimental test between the configurational and feature-interference models. The noise elements form a line, and the target is either in line with them or not. The configurational hypothesis predicts performance will be worse when the target is in the line than when it is out of it, and the feature interference hypothesis predicts, if anything, worse performance when it is out of line than when it is in line with the noise. Experiment 2 always has the target physically out of the line formed by the noise items, but the rules Prytulak formulated are used to vary its degree of grouping with the line. Experiment 3 collects ratings from an independent group of subjects to make sure that Prytulak's rules hold for our configurations.

\section{EXPERIMENT 1}

\section{Method}

Figure 1 illustrates the stimuli used in this experiment. All the configurations had a line of noise elements (hybrid F-T's) and one target $(F$ or $T)$. The target was either in line with the noise elements, as in Figure 1a, or out of the line, as in Figure $1 \mathrm{~b}$. When the target was in line, it was always at the same end of the display as the out-of-line noise item. There were always six elements arranged as in Figure 1, and the configuration had the eight different orientations that result from all four 90 -deg rotations of it, plus all reflections. Thus, the line was either 135 or $45 \mathrm{deg}$ from horizontal, the target could be at either extreme of the array, and the out-of-line item could be on the top or the bottom of the line. The configurational hypothesis predicts that performance will be worse when the target is in the line (Figure la) than out of it (1b), because in the line it is perceptually grouped with the highly confusable noise items. The only prediction that a featureinterference approach could make would be to predict worse performance for configurations like that in Figure $1 \mathrm{~b}$ because there the target has a smaller mean distance from noise than in Figure 1a.'

Procedure. Subjects were briefly shown arrays like those in Figure 1 and had to decide, while timed, whether they contained an $F$ or a $T$. They were asked to respond as quickly as was consistent with accuracy. They saw a fixation point before the stimuli were presented, and the target was always the same distance from the fixation point, no matter what the configuration. There were a total of 32 different stimuli, since there were two targets (F or $T$ ), eight orientations of the configuration, and two target conditions (in or out of the line), and these were combined orthogonally.

The stimuli appeared in a computer-controlled display on a cathode ray tube with a P7 phosphor and were viewed through a blue filter to eliminate the long persistence phosphor trace. The subjects saw the display through a view tunnel at a distance of approximately $57 \mathrm{~cm}$. All the stimulus items subtended a visual angle of approximately 1 deg vertically, and all except the $F$ character subtended a visual angle of $1 \mathrm{deg}$ horizontally. The $F$ subtended a visual angle of $45 \mathrm{~min}$ horizontally. The distance between the centers of adjacent items were approximately $1 \mathrm{deg} 25 \mathrm{~min}$, and the distance from the target item to the center of the visual field was always a visual angle of $4 \mathrm{deg} 7 \mathrm{~min}$.

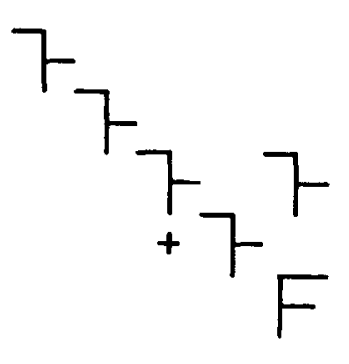

a

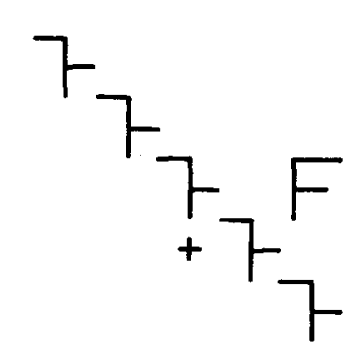

b
Figure 1. Examples of the stimulus patterns in Experiment 1. In pattern $a$, the target groups with the noise items, in pattern b, the target does not group with the noise items. The fixation cross is shown in its position relative to the array, but was not shown while the array was on. 
Each subject participated in two 45-min sessions. In each session, the subject saw a dim fixation dot in the center of the field. After $2 \mathrm{sec}$, the fixation dot dimmed slightly as a ready signal. Five hundred milliseconds following the ready signal, the fixation dot was replaced by the stimulus array. The stimulus array remained on for $50 \mathrm{msec}$ on all experimental trials. A clock started with the offset of the display. The clock was stopped when the subject passed one or two telegraph keys. Half the subjects used the preferred hand to indicate they saw a $T$ target and the other hand to indicate they saw the F, and half the subjects had the opposite assignment. The subject was given feedback immediately after the response, with the words "correct" or "wrong" appearing on the screen for $1 \mathrm{sec}$.

On each day, the subjects received 3 blocks of 32 practice trials, a 5-min rest, and then 10 blocks of 32 experimental trials. The 32 stimuli in each block were presented in a different random order.

Subjects. Ten subjects, seven female, ages 16 to 21 , were recruited through a newspaper advertisement. They were paid $\$ 4$ for their participation. All subjects had visual acuity within the normal range.

\section{Results and Discussion}

Correct reaction times (RTs) were analyzed with target ( $T$ vs. F), target position, and target in vs. target out of the line of noise items as factors in an analysis of variance. The latter factor, the one of central importance, was significant, $F(1,9)=13.27$, $\mathrm{p}<.01$. The average $\mathrm{RT}$ when the target was part of the line was $536 \mathrm{msec}$, and it was $510 \mathrm{msec}$ when it was not part of the line. Thus, good continuation affected performance in the predicted manner.

Several other effects also turned out to be statistically reliable. The $F$ was identified faster than the $T$ (512 vs. $534 \mathrm{msec}$ ), with an $F(1,9)=9.8, \mathrm{p}<.025$. The position of the target in the field also affected RT significantly. The $F(7,63)$ for differences among the eight positions was $3.13, p<.01$. The form of this effect was such that the top two and bottom two positions were processed faster than the left two and right two in the octagonal set of possible target positions. This unusual position effect may be the result of the different configurations (45-deg vs. 135-deg lines) associated with each target position. The configural variable (target in vs. out of line) was perfectly counterbalanced over the eight possible positions in the field, however. Finally, there was an interaction between the configurational effect and target position, $F(7,63)=4.37, p<.01$. The form of the interaction was such that harder positions showed more of a configural effect.

The error rate was $5.3 \%$ overall. The productmoment correlation between error and RT over conditions was .14 and the mean RT of errorful responses was $486 \mathrm{msec}$.

\section{EXPERIMENT 2}

In Experiment 1, a perceptual unit was defined as a line of items. The Gestalt principle of good continuation was used to justify this grouping. Experi- ment 2 partially replicates, and extends, the findings in Experiment 1 by manipulating the strength of good continuation grouping. Good continuation grouping does not require that items be physically in the same straight line, and there are some advantages to using arrays in which they are not. First, if both a target and a noise item are "off" the line in every array, a serial search strategy that starts by inspecting the "off-line" item cannot account for the configurational effect. (As mentioned in the Discussion, this strategy does not avoid a configurational explanation, anyway.) Second, use of offline elements in good continuation allows degree of good continuation grouping to be varied.

The configurations in this experiment use two principles formulated by Prytulak (1974). Figure 2 shows the stimuli to which these principles were applied. In each of the arrays in Figure 2, there is a "shaft" of items and two "arms," and Prytulak's principles tell which arm will group more strongly with the shaft. The first principle is rectilinearity: The arm that comes closest to forming a 180-deg angle with the shaft will group more strongly with it. The second principle is that of orientation: If the shaft is either horizontal or vertical, an arm at $90 \mathrm{deg}$ to it will tend to group with it. In some cases, of course, these two principles will conflict, producing an intermediate degree of grouping for both arms.

In the present experiment, there was always a line of five noise items and two out-of-line items, one of which was the target. One of the two out-of-line items formed a 150-deg angle with the shaft (we will call this arm a) and one formed a 90-deg angle (arm b). The two arms were either on the same side of the shaft, as in Figures $2 a$ and $2 b$ (this will be termed the ipsilateral condition), or on opposite sides, as in Figures $2 \mathrm{c}$ and $2 \mathrm{~d}$ (contralateral condition). The principle of rectilinearity predicts that arm a will tend to group more strongly with the shaft than arm $b$. When the shaft is either vertical or horizontal (as in Figure 2a and $2 c$ ), the principle of orientation predicts that arm $b$ will group with the shaft. Because Prytulak's stimuli were somewhat different from ours, we could not tell whether the principle of orientation, when it applied, would overcome the principle of rectilinearity or merely reduce its effect. Thus, our predictions were based on the

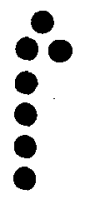

a

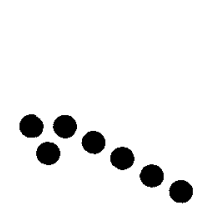

b

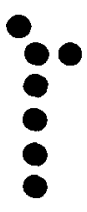

c

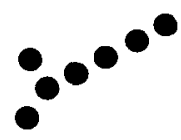

d
Figure 2. Examples of the stimulus patterns in Experiment 2. 
assumption that arm a would group better with the shaft than arm b except when the shaft was horizontal or vertical. In these cases, the grouping of arm a with the line would be reduced. The prediction is, then, that performance will be worse when the target is in position a than when it is in position b, except when the shaft is either horizontal or vertical, in which case the difference between performance when the target is at a vs. at $b$ will at least be reduced, and it may be reversed. The feature-analytic approach could only predict that performance will always be worse with the target in arm $b$ than in arm a for the same reason as in Experiment 1.

A judgment task was given to each subject in addition to the detection task. This was done to assess the grouping structure of each configuration. Experiment 3 assesses the grouping structure with a larger sample of subjects.

\section{Method}

Stimuli. In both the detection experiment and the judgment task, there were 24 stimulus patterns. The 24 patterns can be derived from all 60 -deg rotations and reflections of the patterns in Figures $2 \mathrm{a}$ and $2 \mathrm{c}$. In the judgment task, the stimuli consisted of seven solid circles, $1 / 4 \mathrm{in}$. in diam, printed on white $3 \times 5$ in. index cards. The distance between the perimeters of adjacent circles was $1 / 8$ in. In the detection task, the stimulus patterns consisted of seven circles presented on a cathode ray tube in a computer-controlled display. The noise items in each array were circles with a vertical line passing through the center. The target was a circle that had a vertical chord to the left or right of the center of the circle (see Figure 3), and the subject's task was to decide whether the target with the chord on the left or on the right had been presented.

There were 96 different stimuli in the detection task. This is because, in each of the 24 different configurations, the target could be in arm a or arm b, and it could be a left- or a rightchord target $(96=24 \times 2 \times 2)$.

Stimuli were positioned on the cathode ray tube such that all targets, and both arms in any array, were always the same distance from the center of the display (see Figure 3). The distance between the center of the display and the centers of the circles on each arm subtended a visual angle of $4 \mathrm{deg} 30 \mathrm{~min}$. The diameter of the circles subtended a visual angle of $1 \mathrm{deg}$. The distance between the centers of adjacent circles subtended a visual angle of $1 \mathrm{deg} 30 \mathrm{~min}$. The chord in the target item was offset from the center of the circle a distance of approximately $15 \mathrm{~min}$, either to the left or the right.

Procedure. Half of the subjects received the judgment task first, and half received the detection task first. The procedure in the detection task was the same as in Experiment 1 with the following exceptions. The stimulus remained in view for $100 \mathrm{msec}$ rather than $50 \mathrm{msec}$. The subjects received feedback only when they made an incorrect response. The subjects responded by pressing a left- or right-hand telegraph key for left- and right-of-center target chords, respectively. The detection task took five 45-min sessions. Each session consisted of 30 warm-up trials, a 5-min rest, two blocks of 96 trials, a 5-min rest, and finally two more blocks of 96 trials. The first session was considered practice, and its results are not included in the analysis.

In the judgment task, the subjects were asked to indicate which of the two out-of-line circles "belonged" most to the line of dots. The 24 stimulus cards were shown one at a time in a different random order to each subject. The subjects simply indicated to the experimenter which of the out-of-line dots belonged most to the line of dots. This task was self-paced and took about $5 \mathrm{~min}$.

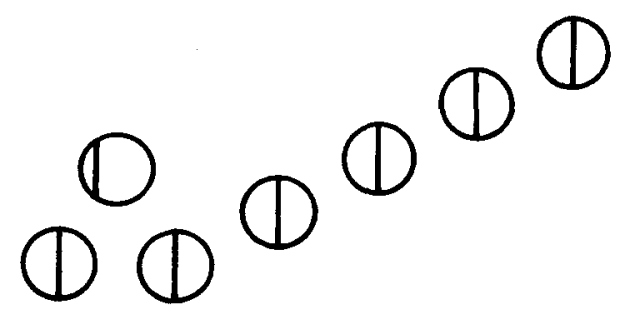

Figure 3. A sample stimulus from the forced-choice detection task in Experiment 2.

Subjects. Eight subjects, six female, ages 15 to 23 , recruited as in Experiment 1, were paid $\$ 10$ for their participation. A ninth subject was eliminated after the first day because his accuracy on the detection task was not above chance.

\section{Results}

Detection task. The analysis of variance of correct RTs in the detection task had target type (left vs. right chord), display type (ipsilateral vs. contralateral arms), and position of target (in arm a vs. in arm b) as orthogonal factors. A fourth factor, nested in the ipsilateral vs. contralateral variable, had 12 levels, each consisting of one of the possible rotations or reflections of either the ipsilateral or contralateral display type. The 12 levels result from the six rotations of $60 \mathrm{deg}$, times the two possible reflections. This fourth factor will be referred to as the rotation/ reflection variable. The grouping principle of rectilinearity overall predicted RT quite well. Arm types a and $b$ had mean RTs, over all configurations, of 666 and $609 \mathrm{msec}$, respectively. This difference was reliable, $F(1,7)=45.03, p<.01$.

The rotation/reflection variable had a significant effect, with $F(22,154)=3.82$, p $<.01$. Furthermore, there was an interaction between position of target (arm a vs. arm b) and rotation/reflection, $F(22,154)=2.98, p<.025$. This interaction followed exactly the pattern expected by the configurational approach, assuming that the principles of orientation and rectilinearity each contribute to the grouping. To show the configurational predictions most clearly, we divided the stimuli into three groups. In Group 1, rectilinearity and orientation made the same prediction (see Figure 2b), in Group 2, only rectilinearity made a prediction at all (see Figure $2 \mathrm{~d}$ ), and in Group 3, rectilinearity and orientation made opposite predictions (see Figure $2 a$ and $2 c$ ). There were 32 stimulus patterns in each set. The difference in RT between arm types (arm a - arm b) was 101, 63, and $7 \mathrm{msec}$ for the stimuli in Groups 1, 2, and 3, respectively. Analysis of variance using the above stimulus grouping as a main effect showed a significant effect of groups, $F(2,14)=21.14, p<.01$. This breakdown of the stimuli into three groups accounts for $50.0 \%$ of the variance of the means in the inter- 
active between array rotation/reflection and target position.

The average RT for ipsilateral displays was $665 \mathrm{msec}$, and it was $610 \mathrm{msec}$ for contralateral displays. This effect is reliable, with an $F(1,7)=$ $75.2, \mathrm{p}<.01$. This effect is predicted by both the configurational approach and the feature-interference approach. The configurational approach predicts it by greater proximity grouping in the ipsilateral case, and the featural approach predicts it by interference due to proximity.

There was a greater effect of good continuation when the target was on the ipsilateral side than when it was on the contralateral side, $F(1,7)=10.65, p<.025$. On the ipsilateral side, detection of a target in arm a was 71 msec faster than in arm b, but on the contralateral side, the advantage of arm a was only $42 \mathrm{msec}$. The final significant effect was a three-way interaction of rotation/reflection, target position, and target type, $F(22,154)=14.00, p<.01$, that defies understanding.

There was no evidence of response competition. When the target was on the same side of the display as the hand that was to make the correct response, the mean RT was $638 \mathrm{msec}$. When it was on the opposite side, and therefore might be expected to cause response competition, the mean RT was $628 \mathrm{msec}$.

The correlation between RT and errors over the 96 stimuli in the detection experiment was $r=.70$. The average percent error was $11.3 \%$ and the mean RT for the errors was $746 \mathrm{msec}$.

Judgment task. For every stimulus card, the majority of subjects chose arm a as grouping most with the line. On 14 cards, all eight subjects unanimously chose arm a as grouping better than $b$ with the line. On nine stimuli, seven out of eight subjects chose arm a, and on 1 card, five out of eight subjects chose arm a. Overall, the rule of rectilinearity predicted grouping just as it did in the detection task. In four out of the eight stimuli where one or more subjects chose arm b as grouping most with the line, rectilinearity and orientation made opposite predictions, and in the remaining four, rectilinearity and orientation made the same prediction. These judgments should not be looked at too closely, however, because there are only a small number of data. Each of only eight subjects made one judgment for each stimulus pattern, compared to 16 RTs per condition per subject in the detection task. The purpose of Experiment 3 was to provide a more reliable independent measure of grouping.

\section{EXPERIMENT 3}

\section{Method}

Stimuli. The stimulus patterns included those in the judgment task of Experiment 2 plus 12 new patterns. These new patterns consisted of a shaft of five dots and two arms. In these new patterns, arm a was $90 \mathrm{deg}$ to the shaft and arm b was $60 \mathrm{deg}$ to the shaft. The two arms were always on opposite sides of the shaft. As is the case with the other stimuli, the six 60 -deg rotations of the pattern plus the two reflections make 12 stimuli. In this third set of patterns, the effect of rectilinearity should be weak, and if orientation predicts grouping it has a better chance of doing so in this set of stimuli. Furthermore, the inclusion of this set of patterns was expected to prevent subjects from establishing a set of always responding intentionally on the basis of rectilinearity, since this set should reduce the obviousness of the principle of rectilinearity.

Stimuli were the same size and dimensions as in the previous judgment task. Each pattern was dittoed on a $3 \times 5$ in, page. In the top right corner of each page was a number from 1 to 36 that identified the stimulus pattern. On the bottom right corner of the page was the letter $S$ and a blank on which the subject's number was recorded after the experiment. Pages were arranged in a different random order for each booklet and, together with an instruction page, stapled in the top left corner.

Subjects. Forty students in an introductory psychology class at Pomona College served as subjects. Two of these subjects did not follow instructions and their data were discarded, leaving 38 subjects in the sample.

Procedure. Booklets were distributed and the instructions were read by the experimenter. The subjects were asked to circle the one out-of-line dot on each page that appeared to group most strongly with the line of dots. When all subjects had finished this task, they were asked to go through the booklet again and on each page write a " 1 " if the grouping was weak, a " 2 " if the grouping was moderate, and a " 3 "' if the grouping was strong. The experiment took about $10 \mathrm{~min}$.

\section{Results}

The data were recorded on a scale from 1 to 6 . In the new scale, a pattern scoring 1 would be one in which all subjects chose $b$ as grouping very strongly with the line, and 6 would imply that a grouped very strongly with the line. The 3-point scale was thus converted into a 6-point scale of the strength of a's grouping, that is, of rectilinearity grouping. An average rating above 3.5 for a configuration indicates that arm a groups better with the line than arm $b$. The three types of starting configurations, ipsilateral and contralateral displays plus the new set of displays, will be referred to as Stimulus Sets 1, 2, and 3 , respectively. The mean rating for Sets 1, 2, and 3 were $5.04,5.01$, and 4.00 , respectively. These means were all significantly higher than 3.5 , with, respectively, $t(37) \mathrm{s}$ of $7.83,6.58$, and 3.47 , all ps $<.01$.

The more general claim that perceptual grouping is a strong determinant of detection performance was assessed in the following manner. A nonparametric correlation, Kendall's tau, was computed between RT and the grouping measure over the 96 stimuli. The RT for configurations with the target position in arm a were paired with the corresponding judgment score (1 to 6), and the RT for configurations with the target position in arm $b$ were paired with the negative of the judgment score $(-1$ to -6$)$. Over the 96 pairs, $\tau=.25$. This correlation is significantly different from zero, $Z=3.56, p<.01$. As was found by Banks and Prinzmetal, judgments of 
perceptual grouping predicted detection performance.

One important difference between the grouping judgments and detection performance, however, was an apparent lack of an effect of the principle of orientation in the judgments. Compared to rectilinearity, orientation seemed to have no effect at all. The test of the relative strengths of the principles of rectilinearity and orientation began by dividing the stimuli into three groups, as was done in Experiment 2 . In Group 1, rectilinearity and orientation made the same predictions, in Group 2, orientation made no prediction, and in Group 3 rectilinearity and orientation made opposite predictions. The mean grouping ratings for the three groups over all three stimulus sets were $4.50,4.79$, and 4.71 for Groups 1, 2, and 3, respectively. These grouping scores increase as arm a groups better with the line, relative to the strength with which $b$ groups with the line. If orientation had any effect on grouping, these scores should have declined monotonically from Group 1, to Group 2, to Group 3. The effect of the principle of orientation seems not to show up at all. This is perhaps not surprising since the orientation of the booklet relative to the subject's frame of reference or retinal orientation was not controlled, as it was in Prytulak's study.

\section{DISCUSSION}

Detection performance in Experiments 1 and 2 was predicted by the Gestalt principle of good continuation. The effect of the configurational variables on grouping was generally confirmed in Experiment 3, although we did not replicate Prytulak's principle of orientation. The results cannot be explained in terms of channel inhibition, feature confusion, or item proximity. The conclusion that the effect of good continuation is the result of perceptual organization seems inescapable.

At this point, it seems that two theoretical issues need to be addressed. First, a mechanism whereby perceptual organization effects performance must be specified. Second, a model of the perceptual parser itself is needed. Three models that operate on the output of the perceptual parser and explain the effect of good continuation will first be discussed. These models are a serial processing model, a parallel attention allocation model, and a model that is based on recursive reparsing of the array.

In the serial processing model, subjects in Experimental 1 would locate the out-of-line item and process that item before any others. This explanation at first glance may not seem to require a perceptual parser at all, but some process has to define a "line" of items before the out-of-line item can be selectively attended. Experiment 2 poses a problem for this theory because there were two out-of-line items. The rule to process the out-of-line item first would not work, but the configural effect was still found. The possibility of serial processing is, of course, not excluded by this experiment, but serial processing must begin after the line of items is defined perceptually.

A second possible model is the attention allocation theory of Kahneman (1973; Kahneman \& Henik, 1976; also cf. Rumelhart, 1970). In this theory, perceptual units are first defined by a unit formation stage. Attention (or, in other terms, processing resources) is then allocated to units. The processing resources allocated to a unit are shared equally by all items in that unit. Individual items in small units will thus receive more processing resources than items in large units. In the present study, the out-ofline item will receive more processing resources than the in-line item because the resources allocated to the other unit (i.e., the line) must be divided between all the elements in the unit.

In the third model, the function of the perceptual parser is to create a perceptual code that is appropriate for subsequent processing. For example, if an item is to be correctly recognized, the perceptual parser must correctly segregate that item from other items before recognition takes place. In the detection experiment, if the parser correctly segregates the target item from the background, then the target can be quickly identified. If the parser groups the target with the noise items, then the resulting unit cannot be recognized as a target item. In this case, the input must be parsed again so that the target is appropriately segregated. This reparsing takes time, and it explains why there is a longer latency when primitive organizational principles (similarity, good continuation, etc.) lead to inappropriate parsing. If the sensory information decays before the display is appropriately organized and the target recognized, the subject must guess. If sensory information only partly decays before the display is appropriately organized, then the subject will guess with a higher than chance probability of being correct.

A choice between these theories cannot be made from the present study. All three formulations depend on the output of a perceptual parser that delivers a structural analysis of visual input. The key to understanding certain detection phenomena therefore would appear to be a model of the perceptual parser. The difficulty of this task is attested to by the almost complete lack of psychological models in this area. We have no new proposals to advance, but some comments within the context of this study can be made.

Gestalt psychology has left us a legacy of a hundred or so organizational principles (Helson, 1933), but no compelling processing model. In the present study we appealed to two principles, rectilinearity and 
orientation. An investigation into the parser might begin by determining whether rectilinearity and orientation, for example, involve the same mechanism. If parsing principles involve different mechanisms or operate at different levels, then they should have different processing consequences. The organizational principles called similarity and proximity grouping could be the result of featural interaction in something like Estes' interactive channels theory. Good continuation grouping, on the other hand, could not be explained by that mechanism. If similarity and proximity grouping are the result of perceptual feature analysis, but good continuation is not, there should be observable processing consequences of this difference.

Broadbent (1977) has recently proposed a mechanism for Gestalt-like organizational effects that does not require a structural perceptual parser. He proposes two recognition systems that are sensitive to different spatial frequencies. One system responds to low visual spatial frequencies and would, in effect, respond to Gestalts in the array while the other system responds to high spatial frequencies and processes feature information. Broadbent's proposal is an attractive alternative to complex parsing mechanisms for explaining configurational effects, but it remains to be seen whether all Gestalt organizational principles can be reduced to a low spatial frequency component. In the present case, however, good continuation grouping does appear to represent a lowfrequency component.

The present results suggest that feature analysis does not come before configurational analysis. If feature analysis preceded configurational analysis, the feature information alone would be enough for subjects to respond and there would be no configurational effect. This conclusion does not depend at all on considerations of spatial frequency, but spatial frequency analysis would also suggest it since, as Broadbent (1977) notes, the low-frequency analysis (which extracts the Gestalt information) generally works faster than the high-frequency analysis (which extracts featural information).

The configurational hypothesis has been used to explain the effect of good continuation, the display size effect, the effects of target-noise similarity and proximity, and the effect of noise homogeneity without reference to channels or feature detectors. This does not mean that there are not feature detectors. However, this study has demonstrated detection phenomena that clearly cannot be accounted for by simple interactions between feature detectors.

\section{REFERENCES}

Banks, W. P., Bodinger, D., \& Illige, M. Visual detection accuracy and target-noise proximity. Bulletin of the Psychonomic Society, 1974, 2, 411-414.

Banks, W. P., \& Prinzmetal, W. Configurational effects in visual information processing. Perception \& Psychophysics, $1976,19,361-367$.

Broadbent, D. E. The hidden preattentive process. American Psychologist, 1977, 32, 109-118.

Eriksen, C. W., \& Eriksen, B. A. Effects of noise letter upon the identification of a target letter in a nonsearch task. Perception \& Psychophysics, 1974, 16, 143-149.

EsTES, W. K. Interactions of signal and background variables in visual processing Perception \& Psychophysics, 1972, 12, 278-286.

Estes, W. K. Redundancy of noise elements and signals in the visual detection of letters. Perception \& Psychophysics, 1974, 16, 53-60.

Estes, W. K., \& TAYloR, H. A. Visual detection in relation to display size and redundancy of critical elements. Perception \& Psychophysics, 1966, 1, 9-16.

GARDNER, G. T. Evidence for independent parallel channels in tachistoscopic perception. Cognitive Psychology, 1973, 4, 130-155.

Helson, H. The fundamental propositions of Gestalt psychology. Psychological Review, 1933, 40, 13-32.

Kahneman, D. Attention and effort. Englewood Cliffs, N.J: Prentice-Hall, 1973.

KahNeman, D., \& HeniK, A. Effects of visual grouping on immediate recall and selective attention. In S. Dornic (Ed.), Attention and performance VI. Hillsdale, N.J: Erlbaum, 1976.

Kinchla, R. A. Detecting target elements in multi-element arrays: A confusability model. Perception \& Psychophysics, 1974, 15, 149.158.

McINTYRe, C., Fox, R., \& Neale, J. Effects of noise similarity and redundancy on the information processed from brief visual displays. Perception \& Psychophysics, 1970, 7, 328-332.

Mclaughlin, J. P., Masterson, F. A., \& Herrmann, D. J. Pattern redundancy and detection in very short term memory. Perception \& Psychophysics, 1972, 12, 205-208.

PRYTUlak, L. S. Good continuation revisited. Joumal of Experimental Psychology, 1974, 102, 773-777.

RUMELHART, D. E. A multi-component theory of the perception of brief visual displays. Journal of Mathematical Psychology, $1970,7,191-218$.

\section{NOTE}

1. The configurational hypothesis can, of course, also make predictions on the basis of target noise proximity because of proximity grouping. However, if another grouping principle, in this case good continuation, has a stronger influence on stimulus organization, then proximity will have little effect on performance. To avoid circularity in deriving predictions from the configurational approach, an independent assessment of grouping is absolutely necessary. This was done in Experiment 3.

(Received for publication December 20, 1976; accepted for publication February 28, 1977.) 